\title{
MIGRATION, FLUCHT UND BEWEGUNG. (KON-)TEXTE ZUM ORTSWECHSEL IM DENKEN
}

\author{
VORWORT DER HERAUSGEBER \\ TOBIAS AKIRA SCHICKHAUS (BAYREUTH) \\ UND ŠTĚPÁN ZBYTOVSKÝ (PRAG)
}

Böhmen liegt am Meer - Ingeborg Bachmann bringt 1966 in ihrem Gedicht ein ,Ich zum Ausdruck, dessen Existenzbedingungen im Ungewissen liegen und dabei sorglos „unverloren“ (V. 12) scheinen: „Grenzt hier ein Wort an mich, so laß ich's grenzen“ (V. 5) und „[i]st Liebesmüh in alle Zeit verloren, verlier ich sie gern.“ (V. 3) Die inhaltliche Eigenheit ihrer Verse betont Ingeborg Bachmann durch eine Zweiteilung in jeweils einen Konditional- und Hauptsatz, also in der Kausalität von Voraussetzung und Folge. Das Versmaß wird durch den strengen Alexandriner getragen, bis Rhythmus sowie Sprechhaltung in der Mitte des Gedichts aufgebrochen werden:

Kommt her, ihr Böhmen alle, Seefahrer, Hafenhuren und Schiffe unverankert. Wollt ihr nicht böhmisch sein, Illyrer, Veroneser und Venezianer alle. Spielt die Komödien, die lachen machen.

Die Strenge des Alexandriners ist nun in Gänze aufgegeben, ebenso die kausal analytische Zweiteilung von Ursache und Folge in der syntaktischen Form; stattdessen greifen die Verse durch Enjambements ineinander und entwickeln eine auffällige über-setzende Dynamik von Grenzenlosigkeit. Das lyrische Ich unterbricht zum ersten Mal seine Selbstreflexion und wendet sich nach außen. Auf dem Grunde des Meeres geht es „zugrund“ und weiß dennoch: „[I]ch bin unverloren“ (V. 12). „Böhmen“ kann als ein Ort-im-Zwischen betrachtet werden, an dem sich Denkstile vielfältiger Kulturen treffen. ,Böhmen' kann aber auch mit der Vorstellung einer Landkarte verbunden werden, worin vielfältige Sprachen eingetragen sind und eine Netzstruktur des interkulturellen Dialogs entstehen lassen. Und schließlich kann ,Böhmen' als Raum erscheinen, dessen kulturelle Grenzen, Zentren/Peripherien und sonstige Konstellationen im Laufe ständiger Austauschprozesse und Auseinandersetzungen fortwährend ein- und übertragen werden, als Raum, in dem Intra- und Interkulturelles eins sind. In diesem Sinne lässt sich etwa auch die Selbstpositionierung Johannes Urzidils als „hinternational“ (Urzidil 1960: 11) verstehen, oder die von Egon Erwin Kisch, wenn er für seine Prager Kindheit die im nationalen Sinne territorialisierende Bezeichnung „Gassenbube“ zurückweist und sich und seinesgleichen „Durchhäuserbuben“ nennt, denn „[u]nser Sinnen geht nur dahin, ein Hintertürchen zu entdecken, durch das sich plötzlich der Eingang in eine neue Welt öffnet“ (Kisch 1920: 73). Böhmen in Bewegung - das sind zuallererst Menschen in Bewegung. 
50 Jahre nach Bachmanns Gedicht kommen die ,Bohemians‘ wieder vom (oder ans) Meer, denn mit dem Tagungstitel Vielfältige Konzepte - Konzepte der Vielfalt: Interkulturalität(en) weltweit setzte die Gesellschaft für interkulturelle Germanistik (GiG) mit ihrer 20. Tagung in Ústí nad Labem und Prag den Akzent auf Akzente des Interkulturellen.

Dem liegt zugrunde, dass gerade die breite Fächerung der wissenschaftlichen Fragestellungen und Zugänge es der interkulturellen Germanistik erlaubt, vielfältige Konzepte weltweit nicht nur zu apostrophieren, sondern tatsächlich in den Blick zu nehmen. Die wissenschaftliche Leitung hatten Dr. habil. Renata Cornejo (Ústí nad Labem), Prof. Dr. Manfred Weinberg (Prag) und Prof. Dr. Gesine Lenore Schiewer (Bayreuth) inne. Renata Cornejo und Manfred Weinberg hatten auch die aufwändige Organisation und Veranstaltung vor Ort der bislang größten GiG-Tagung mit fast 200 Vortragenden aus 48 Ländern und von allen fünf Kontinenten in ihren Händen. Das enorme wissenschaftliche Potenzial des interkulturellen Spektrums bildete sich deutlich in der Vielfalt der fünfzehn Fachsektionen ab, an denen internationale Fachvertreterinnen und -vertreter, etablierte und Nachwuchsforscherinnen und -forscher sowie Promovierende mitwirkten. Die Tagung trug der Überzeugung Rechnung, dass ,seinsgebundene' Grenzen fachdisziplinärer Perspektiven vor allem vermittels seinverbundener ' Zusammenarbeit ertragreich überdacht werden. Dies gelang in den Bereichen der Interkulturellen Literatur- und Medienwissenschaft, Chamisso-Literatur, Interkulturellen Linguistik, Mehrsprachigkeitsforschung, Beforschung Böhmens und Mährens, Historischen Kulturwissenschaft, Didaktik, Theoriebildung der DaF-/DaZ-Forschung, Translationswissenschaft, Bildungsforschung, Dialog- und Konfliktforschung sowie der Beforschung von Heimat und Vertreibung und der Mediävistik.

Die in diesem Band versammelten Beiträge sind eine Auswahl dieser Konferenz und widmen sich aus literatur-, sprach- und kulturwissenschaftlicher Perspektive dem Umgang mit Migration, Flucht und Bewegung im Ausgang von gesellschaftspolitischen, spracherwerbstheoretisch geleiteten und literaturwissenschaftlichen Ansätzen.

Um es gleich vorwegzunehmen: an dem hier skizzierten Problemhorizont ist wahrlich nichts völlig Neues. Nur allzu gut sind uns die passionierten Zweifel bekannt, ob Großunternehmungen namens ,Globalisierung' eine internationale Vernetzung befördern oder eine seelische Verletzung am Menschen verursachen; ob die globale Verlagerung von Arbeitsplätzen automatisch ihre Vernichtung beschleunigt oder ob grenzüberschreitende Migrationsbewegungen weltanschaulichen Grenzen identitärer Bewegungen Nahrung geben. Kurzum: ob die janusköpfige Perspektive auf das Feld der Grenzüberschreitung schlussendlich nur einer weiteren fragmentarisierten Blockade im Denken Vorschub leistet.

Nein, die vorliegenden Beiträge, die man in ihrer Komposition - und im Tynjanow'schen Sinne (vgl. Tynjanow 1971) - guten Gewissens auch als ,Reihe' bezeichnen darf, gehen in ihrer außerliterarischen Funktion weiter. Sie spüren in sowohl literarisch-thematischer als auch sprachlich-formaler Hinsicht nicht nur dem aktuellen Umgang mit diesen Fragen nach, sondern bilden in einem erfreulich breit gefächerten Kaleidoskop auch die historisch gewachsenen Bedingungen von interkulturellen Migrationsdiskursen ab. Hierbei zeichnet sich ein thematischer Rhythmus ab, der sich auch wieder mit dem eingangs erwähnten Gedicht von Ingeborg Bachmann gut erklären lässt: Wandert die Sprechhaltung von einer nach innen gekehrten Reflexion zu einem an die Außenwelt gerichteten Appell, so über-setzen auch die folgenden Beiträge in sol- 
che Zugänge, die an der sprachlichen Form interessiert sind, und andere, die primär darauf achten, was über die relational inhaltlichen Verhältnisse ausgesagt wird. Der sich abwechselnde Rhythmus zwischen Text und Kontext steht Pate für die Suche nach Referenzialität um, in und zwischen Migration, Flucht und Bewegung.

„Wohin kannst du gehen, wenn statt eines Ortes eine Person dein Zuhause ist?“ (Mora 2013: 72) Carme Bescansa (Vitoria-Gasteiz) befasst sich im Ausgang von Julia Kristevas Studie Fremde sind wir uns selbst (1990) und im produktiven Austausch mit gegenwärtigen Theorieansätzen von Heimat/Heimatlosigkeit/Nomadismus bzw. Sprache/ Sprachlosigkeit mit der Frage nach Grenzen und Möglichkeiten von Identität jenseits dichotomischer Differenzkonstruktionen. Ihr Beitrag Heterogenität im gegenwärtigen Heimatdiskurs am Beispiel von Terézia Moras Roman ,Das Ungeheuer' nähert sich der Frage anhand einer literaturwissenschaftlichen Re-Lektüre des Romans.

Die Geschichte der Vertreibung ist zweifelsohne fester Bestandteil europäischer Geschichtsschreibung. Der Beitrag von Tomasz Dziura (Wrocław) Erinnerung als Kultur, Kulturen des Erinnerns - Initiativen zum Gedenken im Ausgang deutsch, tschechischer und polnischer Debatten dokumentiert mithilfe eines diskursanalytischen Ansatzes und des Konzepts der Erinnerungsorte Funktionen und Formen der aktuellen Erinnerungskultur und leistet einen wichtigen Einblick in den politischen Umgang mit Flucht und Vertreibung. Schlussendlich geht es um die Frage, ob Erinnerungskulturen fähig sind, die Konfliktivität in der Vergangenheitsbewältigung produktiv für die Gestaltung von Zukunft zu nutzen.

Ähnlich in der Fragestellung, aber anders im Gegenstand lenkt dann Barbara Heinsch (Oviedo) den Fokus von der Erinnerungskultur auf die Kulturvermittlung; genauer gesagt auf die Frage, wie sprachenpolitische Konzepte der Mehrsprachigkeit und jene werteorientierte von Interkulturalität in Einklang zu bringen sind. Nach einer Vorstellung bildungspolitischer Ansätze unternimmt ihr Beitrag eine empirische Evaluation zu Kulturellen Deutungsmustern interkulturellen Handelns und ermittelt daraus ihre Bedeutung für die Grundlagenforschung und Anwendung im DaF-Bereich.

Im Spiegel von Sprachen sitzen multiperspektivische Ansätze interkultureller Schreibweisen. Ihr Potenzial, alternative Sprachsehgewohnheiten auf festgefahrene Doktrinen und Sprachnormen zu fördern, ist Ausgangspunkt zur Untersuchung von „literarischen Entdeckungsreisen multipler Identitäten“ im Beitrag von Christina Markoudi (Thessaloniki) mit dem Titel Herta Müllers ,Worthunger' als Interkulturalitätskonzept.

Alžběta Peštová (Olmütz) „entführt“ uns in das Prag der Jahrhundertwende. Ihr Beitrag Entsagung oder Desillusion? Der Student als Paradigma des Spätrealismus in der tschechischen und deutschen Literatur illustriert am Beispiel der Romane Die Vaclavbude (1902) von Karl Hans Strobl und Santa Lucia (1893) von Vilém Mrštík das weltanschaulich vielschichtige Verhältnis des Prager Studentenlebens im spannungsvollen Prozess deutsch-tschechischer Nationalisierungsbestrebungen, u.a. im Vergleich mit den Stereotypen vom national klar geteilten Prag.

„WILLKommen“! Die preisgekrönte Tragikomödie Almanya - Willkommen in Deutschland (2011) erzählt die Geschichte einer seit drei Generationen in Deutschland lebenden türkischen Migrantenfamilie und ihre gemeinsame Reise in die Türkei. Canan Şenöz-Ayata (Istanbul) untersucht in ihrem Beitrag Ein- oder Mehrsprachigkeit? Analyse der Sprachverwendung und Sprachreflexion im Film, Almanya - Willkommen in Deutsch- 
land' filmische Strategien sprachlicher Verfremdung im Ausgang von Bertolt Brechts Konzept des Epischen Theaters.

Die Themen Flucht und Migration bestimmen aktuell stärker denn je den politischen Diskurs in Europa. Was muss in diesem Zusammenhang eine interkulturelle Fortbildung leisten? Dieser Frage geht Cornelia Springer (Hamburg) in ihrer empirischen Evaluation Interkulturelle Kompetenz für Ehrenamtliche in der Flüchtlingsarbeit auf dem Prüfstand nach. Ihre Studie konzentriert sich dabei auf den Crashkurstrend in der Freiwilligenqualifizierung.

Die Beiträge weiterer Tagungsteilnehmerinnen und -teilnehmer samt der Plenarvorträge sind inzwischen erschienen im Themenheft zur Interkulturalitätstheorie der Zeitschrift für interkulturelle Germanistik (2017/2) und in der elften Nummer des Jahrbuchs Aussiger Beiträge mit dem thematischen Schwerpunkt „Sprachwissenschaft und Fremdsprachendidaktik im Spannungsfeld interkultureller Vielfalt“. Im Druck ist aktuell die 2017er Nummer von Brücken. Germanistisches Jahrbuch Tschechien - Slowakei mit den auf Böhmen und Mähren bezogenen Beiträgen; in der Editionsreihe „Cross-Cultural Communication“ des Peter Lang Verlags wird 2018 ein Band mit Beiträgen aus mehreren Tagungssektionen erscheinen.

Zum Gelingen dieses Sonderheftes haben viele beigetragen: Zunächst sei der Redaktion der Germanistica Pragensia für die Publikation und redaktionelle Unterstützung herzlich gedankt. Zu großem Dank sind wir außerdem den Gutachterinnen und Gutachtern aller Beiträge verpflichtet, die uns während der Gestaltung mit Rat und Tat zur Seite standen sowie natürlich den Autorinnen und Autoren für ihre wertvollen und facettenreichen Beiträge.

Vor allem aber sei den vielen Mitwirkenden der GiG-Tagung 2016 gedankt sowie der Schriftleitung der Tagungsakten und des vorliegenden Bandes, Dr. habil. Renata Cornejo, Prof. Dr. Gesine Lenore Schiewer und Prof. Dr. Manfred Weinberg. Ihr unermüdlicher Einsatz für die Vernetzung interkultureller Germanistiken hat den Kongress 2016 an der Jan-Evangelista-Purkyně-Universität in Ústí nad Labem und an der Karls-Universität in Prag erst ermöglicht.

\section{LITERATUR}

Bachmann, Ingeborg (1966): Böhmen liegt am Meer. In: IX. Festival dei Due Mondi. 24 giugno - 17 luglio 1966. [Programmheft Festival di Spoleto.] Spoleto, S. 27.

Bergerová, Hana / Schiewer, Gesine Lenore / Schuppener, Georg (Hgg.) (2017): Sprachwissenschaft und Fremdsprachendidaktik im Spannungsfeld interkultureller Vielfalt [= Aussiger Beiträge 11]. Wien: Praesens.

Cornejo, Renata / Schiewer, Gesine Lenore / Weinberg, Manfred (Hgg.) (2017): Zeitschrift für interkulturelle Germanistik 8, H. 2: Vielfältige Konzepte - Konzepte der Vielfalt. Zur Theorie von Interkulturalität. Bielefeld: Transcript.

Mora, Terézia (2013): Das Ungeheuer. München: Luchterhand.

Tynjanow, Jurij Nikolajewitsch (1971): Über die literarische Evolution [Orig. 1927]. In: Jurij Striedter (Hg.): Russischer Formalismus. Texte zur allgemeinen Literaturtheorie und zur Theorie der Prosa. München: UTB, $433-461$.

Urzidil, Johannes: Predella. Relief der Stadt. In: Ders.: Prager Triptychon. Erzählungen. München 1960, 7-27. 